\title{
THE
}

\section{Delay in Antibiotic Administration Is Associated With Mortality Among Septic Shock Patients With Staphylococcus aureus Bacteremia}

Keith A. Corl

Zeba

Aisling R. Caffrey

University of Rhode Island, aisling_caffrey@uri.edu

Matthew Hermenau

Vrishali Lopes

Follow this and additional works at: https://digitalcommons.uri.edu/php_facpubs

The University of Rhode Island Faculty have made this article openly available. Please let us know how Open Access to this research benefits you.

This is a pre-publication author manuscript of the final, published article.

Terms of Use

This article is made available under the terms and conditions applicable towards Open Access Policy Articles, as set forth in our Terms of Use.

\section{Citation/Publisher Attribution}

Corl, K. A., Zeba, F., Caffrey, A. R., Hermenau, M., Lopes, V., Phillips, G.,..LaPlante, K. L. (2020). Delay in Antibiotic Administration Is Associated With Mortality Among Septic Shock Patients With Staphylococcus aureus Bacteremia. Clinical Investigations, 48(4), 525-532. doi: 10.1097/CCM.0000000000004212 Available at: http://dx.doi.org/10.1097/CCM.0000000000004212 


\section{Authors}

Keith A. Corl, Zeba, Aisling R. Caffrey, Matthew Hermenau, Vrishali Lopes, Gary Phillips, Roland C. Merchant, Mitchell Levy, and Kerry L. LaPlante 


\title{
Delay in Antibiotic Administration is Associated with Mortality Among \\ Septic Shock Patients with Staphylococcus aureus Bacteremia
}

\author{
Keith A Corl MD, ScMª ; Fatima Zeba MD ${ }^{\text {b; }}$ Aisling R. Caffrey, PhD, MS ${ }^{c, d, e}$; \\ Matthew Hermenau Pharm.D ${ }^{\mathrm{c}}$; Vrishali Lopes $\mathrm{MS}^{\mathrm{c}}$ Gary Phillips MAS ${ }^{\mathrm{f}}$; Roland C Merchant, MD, MPH, \\ $\mathrm{ScD}^{\mathrm{e}, \mathrm{g}}$; Mitchell M Levy, MD ${ }^{\mathrm{a}}$; Kerry L. LaPlante, Pharm.D $\mathrm{D}^{\mathrm{c}, \mathrm{d,h}}$
}

\section{Authors' affiliations:}

Department of ${ }^{a}$ Medicine, Division of Pulmonary Critical Care and Sleep, Warren Alpert Medical School of Brown University, Providence, RI; ${ }^{b}$ Department of Medicine Kent Hospital, Alpert Medical School of Brown University, Warwick, RI; 'Veterans Affairs Medical Center, Infectious Diseases Research Program and Center of Innovation in Long Term Services and Supports, Providence, Rhode Island; ${ }^{\mathrm{d}}$ University of Rhode Island College of Pharmacy, Kingston, Rhode Island; 'Brown University School of Public Health, Providence, Rhode Island; ${ }^{\mathrm{f}}$ Consultant retired from the Center for Biostatistics, Department of Biomedical Informatics, Ohio State University, Columbus, Ohio; ${ }^{\mathrm{g}}$ Department of Emergency Medicine, Brigham and Women's Hospital, Harvard Medical School, Boston, MA; and the hivision of Infectious Disease, Warren Alpert Medical School of Brown University, Providence, RI

\section{Correspondence and request for reprints:}

Keith Corl, MD ScM

Department of Medicine, Division of Pulmonary, Critical Care \& Sleep

The Miriam Hospital

164 Summit Ave., Suite 221

Providence, RI 02906 
Email: keith_corl@brown.edu

Ph: 401-793-4501; Fax: 401-793-4511

Author's Emails: keith_corl@brown.edu; fatima_zeba@brown.edu; aisling_caffrey@uri.edu; mhermenau@my.uri.edu; vrishali.Lopes@va.gov; gphillips.stat@gmail.com; rmerchant@bwh.harvard.edu; mitchell_levy@brown.edu; kerrylaplante@uri.edu

Funding: Dr. Corl was supported by the VA Advanced Fellowship Program in Health Services Research and Development.

Disclaimer: The views expressed are those of the authors and do not necessarily reflect the position or policy of the United States Department of Veterans Affairs.

Conflicts: AC and KL has received research funding from Pfizer, Cubist (Merck, ), and The Medicines Company.

Running Title: Time to Antibiotics in S. aureus Bacteremic Septic Shock Word Count: 3244 


\begin{abstract}
Objectives: The relationship between the timing of antibiotics and mortality among sepsis shock patients has not been examined among patients specifically with Staphylococcus aureus (S. aureus) bacteremia. Design: Retrospective analysis of a Veterans Affairs S. aureus bacteremia database.

Setting: One hundred twenty-two hospitals in the VA Health System.

Patients: Patients with septic shock and S. aureus bacteremia admitted directly from the emergency department to the intensive care unit from January 1, 2003 to October 1, 2015 were evaluated.

Intervention: Time to appropriate antibiotic administration and 30-day mortality.

Measurements and Main Results: A total of 506 patients with S. aureus bacteremia and septic shock were included in the analysis. Thirty-day mortality was $78.1 \%$ for the entire cohort and was similar for those participants with methicillin resistant (MRSA) and methicillin sensitive S. aureus (MSSA) bacteremia. Our multivariate analysis revealed that as compared to those who received appropriate antibiotics within one hour after ED presentation, each additional hour that passed before appropriate antibiotics were administered produced an odds ratio of 1.11 [95\% CI: 1.02-1.21] of mortality within 30 days. This odds increase equates to an average adjusted mortality increase of $1.3 \%$ [95\% CI: $0.4 \%-2.2 \%$ ] for every hour that passes before antibiotics are administered.
\end{abstract}

Conclusions: The results of this study further support the importance of prompt appropriate antibiotic administration for patients with septic shock. Physicians should consider acting quickly to administer antibiotics with $S$. aureus coverage to any patient suspected of having septic shock.

Key Words: Staphylococcus aureus; antibiotics; septic shock 


\section{Manuscript}

\section{Introduction}

Sepsis is a significant global public health burden that afflicts over 31 million people and causes an estimated 5.3 million deaths annually [1]. International guidelines and federal quality measures call for timely administration of antibiotics to septic patients [2,3], and in the case of septic shock, now advise that they be given within the first hour [4].

Initial studies examining the relationship between time to antibiotics and sepsis mortality produced mixed results, but suggest a benefit from early antibiotic administration [5-19]. Two subsequent studies that examined large state-wide databases provide the most compelling evidence that early administration of antibiotics saves lives. A 2017 study of 35,000 septic patients in a California healthcare system showed that delays in antibiotic administration were associated with increased mortality [20]. The association was most pronounced among septic shock patients who suffered a $1.8 \%$ increase in the probability of mortality for each hour antibiotics were not provided. Additionally, an analysis of the New York State Sepsis Initiative revealed that timely completion of a sepsis bundle was associated with reduced mortality among septic patients [21]. Of the sepsis bundle components, early antibiotic administration was the component most strongly associated with the observed decrease in mortality.

Previous studies that evaluated the relationship between the timing of antibiotics and mortality among sepsis patients have, with one exception [19], studied a cohort of patients with sepsis from a variety of bacterial species. To date, the relationship between the timing of antibiotic administration and sepsis morality has not been examined among septic shock patients with Staphylococcus aureus (S. aureus) bacteremia. Both methicillin-sensitive S. aureus (MSSA) and methicillin-resistant S. aureus (MRSA), are a leading cause of sepsis due to skin and soft tissue infections, endocarditis, osteoarticular infections, pneumonia, toxic shock syndrome, and line or medical device infections [22]. MRSA bacteremia is independently associated with sepsis mortality [23, 24]. Among critically-ill patients with MRSA 
bacteremia mortality rates can exceed $60 \%$ [25]. Given the high rate of mortality among septic patients infected with S. aureus, it is important to determine if the relationships observed previously among patients with a heterogenous bacterial sepsis source also hold for those with $S$. aureus bacteremia.

We studied the relationship between the time of appropriate antibiotic administration following emergency department triage and mortality among patients with septic shock admitted to the intensive care unit (ICU) whose blood cultures revealed a S. aureus infection. We then examined the effects that MRSA and MSSA had on our findings.

\section{Methods}

\section{Participants, data source, and data elements}

We conducted a retrospective study of adult patients (age $\geq 18$ years) with $S$. aureus (MRSA or MSSA) bacteremia admitted to 122 hospitals in the Veterans Affairs (VA) Health System from January 1, 2003 to October 1, 2015. We included patients who fulfilled the 2001 International Sepsis Definitions (Sepsis-2) for septic shock [26] using the International Classification of Disease Clinical Modification $9^{\text {th }}$ edition diagnosis codes: 785.52 [27]. We included only those patients who presented to the emergency department (ED) with sepsis, were admitted directly to the intensive care unit (ICU), and received appropriate antibiotics. We excluded patients in whom establishing a "time zero" for antibiotic administration is difficult. This included patients who developed septic shock on a medical or surgical ward, were transferred from an outside hospital, or were directly admitted from an outpatient setting to the hospital. Patients who first received antibiotics more than 12 hours after ED triage were excluded since it is possible that these patients developed sepsis following admission to the ICU (Figure \#1). Limiting the sample to ED patients admitted to the ICU enabled us to focus on those patients for whom a clear and reproducible "time zero" could be identified for tracking the time elapsed prior to antibiotic administration. 
Clinical data for study participants were obtained from the national VA databases through a review of the electronic medical record (EMR) using automated electronic data extraction. Medical history and clinical characteristics extracted from each participant's EMR included: microbiologic and chemistry laboratory data, vital signs, the Acute Physiology and Chronic Health Evaluation (APACHE) III score, and pharmacy data including bar code medication administration records. Trained study personnel then manually extracted time of ED presentation, presence of shock and refractory shock, S. aureus appropriate antibiotic administration, and time from ED presentation to antibiotic presentation from the EMR using established chart review methodology [28]. We created a series of predetermined rules and a procedural manual for data extraction. Two data extractors, blinded to the study outcome, were trained by the lead investigator and required to show competence extracting 20 charts prior to independent data abstraction. Time of ED presentation was defined as the date/time the first ED vital signs were entered into the EMR. Shock was considered to be present if participants had as a systolic blood pressure $<90 \mathrm{mmHg}$, a mean arterial pressure (MAP) $<65 \mathrm{mmHg}$, or if vasopressor agents were initiated. Shock also had to have lasted for more than one hour and be refractory following $1000 \mathrm{ml}$ of intravenous (IV) fluid. If, however vasopressor agents were initiated and were infused for more than one hour, a participant was considered to have refractory shock even if his/her vital signs became normal. In cases for which a participant did not receive either IV fluid or vasopressors, a participant was considered to have refractory shock if he/she had one hour of sustained hypotension (Supplementary Table 1). An a priori list of appropriate antibiotics was created for both MRSA and MSSA bacteremia (Supplementary Table 2). The date/time of the first use of one of these antibiotics was recorded as the antibiotic administration time. For cases in which multiple antibiotics were administered, the date/time of the first appropriate antibiotic recorded in the EMR was used in the analysis. Time to antibiotic administration was calculated as the difference between time from ED presentation to appropriate antibiotic administration. The outcome of interest, all-cause mortality within 30 days of the first positive $S$. aureus blood culture, was electronically extracted from the EMR. 
The Institutional Review Board and Research and Development Committee of the Providence VA Health System approved this study with a waiver of informed consent for patients.

\section{Statistical Analysis}

Participant clinical and demographic characteristics were summarized using descriptive statistics. Chained multiple imputation $(n=20)$ was used to impute missing values for body mass index $(B M I, n=13)$, APACHE III score $(n=4)$, and systemic inflammatory response syndrome (SIRS) criteria present at the time of blood culture $(n=38)$. Fisher's exact and Wilcoxon rank-sum tests were used to assess for differences between survivors and non-survivors for dichotomous and continuous characteristics, respectively.

Logistic regression modeling was used to evaluate the relationship between time of appropriate antibiotic administration and 30-day mortality using the dataset containing the original and the imputed missing variables. Separate models were created for the entire study sample and stratified by participants whose S. aureus was classified as MRSA or MSSA, if shock preceded or followed antibiotic administration, and if shock developed within 12 hours or after 12 hours from ED presentation. Time to antibiotics administration was entered into the model as a linear continuous variable, based on the method of fractional polynomials. We assessed 862 variables representing clinical characteristics including infections source, concomitant infections, APACHE score, medical history and current comorbidities as potential confounders of time to appropriate antibiotic therapy and mortality. Additional variables considered for the model were those believed to have clinical relevance in the relationship of time to antibiotic administration and mortality. Confounders were considered further if they changed the time to antibiotics administration variable coefficient more than $\pm 15 \%$, regardless of statistical significance. Variables were considered to be effect-modifiers if they had a statistically significant interaction with the time to antibiotics administration. A stepwise forward selection process was used to create the final model. Variables were retained in the model if they changed at least one other model coefficient by $\pm 15 \%$. This produced a multivariate model 
adjusted for: index year, BMI, APACHE III score, infective arthritis, SIRS criteria present at the time of blood culture, and MRSA/MSSA. All analyses were performed using Stata/MP version 14.2.

\section{Results}

\section{Participant Characteristics}

We identified 506 study participants with $S$. aureus bacteremia and septic shock who met our inclusion and exclusion criteria (Figure 1), 268 (53\%) with MRSA and 238 (47\%) with MSSA. The median age of study participants was 65 years old (interquartile range [IQR] 58-75), 98.2\% were male, and the median APACHE III score was 66 (IQR 52-81). We observed a significant amount of baseline organ dysfunction with a majority of participants having a concurrent diagnosis of a cardiovascular (91.3\%), pulmonary $(75.5 \%)$, rheumatologic $(74.5 \%)$, hematologic $(66.8 \%)$, central nervous system $(64.2 \%)$, renal (60.9\%), endocrine $(60.1 \%)$, or psychiatric $(59.3 \%)$ process (Table \#1). Details of specific diagnoses that comprised each organ system are outlined in the appendix (Supplementary Table 3). Thirty-day mortality was $78.1 \%$ for the entire cohort, and was similar for those participants with MRSA or MSSA (80.3\% vs. $76.1 \% \mathrm{p}<0.28)$. Univariate analysis showed that survivors were more likely than non-survivors to have a higher BMI, lower APACHE III score, a skin and soft tissue infection as the source of their bacteremia, and to have undergone surgery during hospitalization (Supplementary Table 4).

\section{Antibiotic Administration and Blood Cultures}

The median time to antibiotic administration from triage was 3 hours (IQR 1-5). All participants received an a priori defined appropriate antibiotic. For cases of MRSA, $96.3 \%$ of the participants received vancomycin as the first antibiotic administered with anti-MRSA activity. For cases of MSSA, 40.3\% received vancomycin, $30.5 \%$ piperacillin/tazobactam, $13.9 \%$ ceftriaxone or cefepime, and $10.3 \%$ levofloxacin, moxifloxacin, or ciprofloxacin as the first antibiotic with anti-MSSA activity. Twenty-eight percent of participants received appropriate antibiotics upon arrival or within the first hour of care in the ED; $58.6 \%$ received antibiotics within the first 3 hours of care. The distribution of antibiotic administration 
is displayed in Figure 2. Blood cultures were obtained in $95 \%$ of the participants within the first three days of hospitalization or in the two days preceding hospitalization.

\section{Primary outcome: 30-day Mortality}

Our multivariate analysis revealed that, as compared to those who received antibiotics within one hour following ED presentation, each additional hour that passed before antibiotics were administered produced an odds ratio of $1.11(\mathrm{p}=0.01)$ of 30-day mortality (Table 2 and Figure 3 ). This odds increase equates to an average adjusted mortality increase of $1.3 \%$ [95\% CI: $0.4 \%-2.2 \%$ ] for every hour that passes before antibiotics are administered (Supplementary Table 5). The odds of mortality were not increased for time elapsed to antibiotic administration when participants were stratified by MRSA (OR $1.15 ; \mathrm{p}=0.06)$ or MSSA (OR 1.08; $\mathrm{p}=0.12)$ status, and were similar for participants who developed shock either before (OR 1.14; $\mathrm{p}=0.05)$ or after antibiotic administration $(1.09 ; \mathrm{p}=0.10$; Table 2$)$. However, mortality was greater among the 298 participants who developed septic shock within 12 hours of ED presentation (OR 1.13; $\mathrm{p}=0.03)$.

\section{Discussion}

We examined the relationship between timing of appropriate antibiotic administration and mortality among patients with septic shock due to S. aureus bacteremia in the Veterans Affairs Healthcare System. We found that each elapsed hour between time of ED presentation and antibiotic administration was associated with a $11 \%$ increase in the odds of 30-day morality. This effect was most notable among patients who developed shock within 12 hours after ED presentation.

Our findings enhance previous research on timing of antibiotic administration for patients with septic shock because we specifically focused on patients with $S$. aureus bacteremia. Our study differs from most previous research as we have identified a population where $100 \%$ of the participants have confirmed bacteremia. This dramatically reduces/eliminates the possibility that patients were included in our analyses 
who were presumed to have septic shock but in fact in retrospect were never truly infected. This potential misclassification may have diminished the findings of previous studies who reported an association between the timing of antibiotics and morality and were not able to document bacteremia among all participants. The high 30-day mortality among our study population (78\%) underscores the importance of antibiotic administration timing when $S$. aureus is the culprit organism. The elevated mortality rate reflects the severe acuity of these patients who had a significant amount of concomitant baseline organ dysfunction requiring ICU admission for septic shock. In comparison, in-hospital mortality among septic shock patients with nonbacteremic or bacteremic shock (from a variety of bacterial species) is approximately 51\% [29].

We recognize that antimicrobial culture results and sensitivity data are rarely available when a septic patient first presents to the ED. Therefore, our study findings might only be practically applied for septic shock patients who are suspected to have $S$. aureus bacteremia. Nevertheless, S. aureus is the most common cause of septic shock [23]. When septic shock is believed to be secondary to skin/soft tissue, osteoarticular, endocarditis, and intravenous line or medical device infections, ED physicians will likely empirically administer antibiotics for $S$. aureus; whereas physicians may be less likely to consider a $S$. aureus infection in patients with pulmonary, abdominal or genitourinary infections. In our study, only $39 \%$ of patients had a sepsis source that would likely have been identified during the ED history and physical examination as attributable to $S$. aureus (skin, endocarditis, and osteomyelitis/osteoarthritis), while the majority had a less obvious source of $S$. aureus septic shock (pulmonary or genitourinary). The importance of appropriate antibiotics is well recognized in sepsis and in cases of $S$. aureus bacteremia. Inappropriate antibiotic administration is associated with increased mortality [30]. Sepsis mortality among patients with MRSA bacteremia is greater than mortality for MSSA bacteremia [23-25]. Additionally, inadequate initial dosing of vancomycin for MRSA septic shock is associated with mortality [31]. Our observation of similar mortality rates for MRSA $(80.3 \%)$ and MSSA $(76.1 \%)$ is likely due to the severity of illness among our study population. Our stratified analysis suggests that time to antibiotic administration might be more critical for 
patients with MRSA $(\mathrm{OR}=1.15)$ compared to patients with MSSA $(\mathrm{OR}=1.08)$, although these results did not reach statistical significance. Rapid diagnostic techniques in the ED for $S$. aureus may have an important future role in identifying patients with S. aureus, and in differentiating MRSA from MSSA [32]. Until then, the results of this study suggest that physicians should empirically and rapidly administer broad spectrum antibiotics that include MRSA coverage for any case of suspected septic shock.

Recent work using large healthcare system and state-wide databases has demonstrated an association between antibiotic delay and mortality among patients with sepsis [20,21], with the most robust associations being observed for patients with septic shock. The Surviving Sepsis Campaign (SSC) and the Centers for Medicare and Medicaid Services (CMS) sepsis core measure both call for the prompt administration of antibiotics for all patients with sepsis and septic shock [2, 3]. A recent analysis of septic patients, the majority of whom had received antibiotics within the CMS three-hour window, suggests that even shorter delays in antibiotic administration may be harmful. In their study, administration of antibiotics more than 125 minutes following sepsis identification (shock or SIRS criteria) was associated with increased mortality [33]. However, efforts to administer antibiotics quickly, especially in patients with lower illness severity, must be balanced with potential secondary harms. These harms include renal injury, hepatitis, drug associated rashes, myelosuppression, the emergence of antibiotic resistance [34], and expenditure of scarce ED resources [35]. While using robust methods, even well done analyses of large databases may suffer from unmeasured confounders and confounding by indication. This has led to calls to administer antibiotics as soon as septic shock is identified but adopt a more pragmatic approach towards septic patients where identification of organ dysfunction attributable to sepsis requires more time [36]. Our finding, that each hour delay in antibiotic administration is associated with a $1.3 \%$ increase in mortality among S. aureus septic shock patients is concordant with the septic shock subgroup described by Liu et al. (1.8\%) [20], and suggests that physicians should quickly administer antibiotics in cases of septic shock. 
Our study has limitations. First, the observational study design cannot establish a causal relationship between the timing of antibiotic administration and mortality. Second, the VA population is predominately male, which may limit the external validity of these findings to female patients with S. aureus bacteremia and septic shock. While some data suggest that male patients develop bacteremia at a higher rate than female patients [37], data on gender differences in sepsis mortality are mixed [38-40]. Therefore, we suspect we would observe similar results if this analysis was performed in a predominately female cohort. Third, our observed 30-day mortality rate of $78.1 \%$ is high. As noted previously, we suspect the high mortality is due to the patients selected for the study (S. aureus bacteremia, ICU admissions directly from the ED, multiple baseline organ dysfunction, and septic shock). We observed a statistically significant decline in 30-day mortality from $85.7 \%$ in 2004 to $69.5 \%$ in 2015 (Supplementary Table 6) in the VA hospitals that follows a similar decreasing international trend during a similar time period [41]. Previous estimates of S. aureus bacteremia are less than our observed mortality rates, but in few instances are patients with S. aureus bacteremia and septic shock examined. A 2002 cross sectional observational study of 190 ICUs across Europe found mortality rates of 37 and $42 \%$ for MSSA and MRSA sepsis, respectively, however less than half of these patient had septic shock [23]. Only small case control studies have examined patients with $S$. aureus bacteremia with concurrent septic shock and report mortality rates as high $72 \%$, which is consistent with the results of our study [31, 42]. Our use of ICD-9 diagnosis codes to identify patients with septic shock is subject to misclassification bias. It is plausible that patients with septic shock who died were more likely to receive a formal diagnosis code of septic shock compared to septic shock patients who survived. A strength of our study is that we combine ICD-9 diagnosis with additional clinical variables: positive blood cultures, independent confirmation of shock by chart review, and direct admission from the ER to ICU. Combining ICD-9 diagnosis codes with clinical variables has been shown to increase the sensitivity and positive predicted value of the sepsis diagnosis code $[43,44]$, and the VA coding patterns for serious bacterial infection tend to be highly accurate [45]. Fourth, $41 \%$ of our study population 
developed shock more than 12 hours after triage. In a stratified analysis among this group time to antibiotic administration was not associated with mortality. This suggests that prompt administration of antibiotics may only be beneficial for septic patients who present in, or rapidly develop, shock. Fifth, while stratified analyses suggested that a delay in antibiotic administration was associated with a higher odds ratio of mortality among patients with MRSA and for those patients who develop shock before antibiotic administration, these results did not reach statistical significance. This is likely due to the relatively small sample size for these subgroups, which limited our ability to characterize mortality trends. Further research that focuses on sepsis mortality and antibiotic administration in these populations is needed.

\section{Conclusion}

In summary, among septic shock patients with S. aureus bacteremia admitted directly from the ED to the ICU, we found that each hour delay in appropriate antibiotic administration is associated with an $11 \%$ increase in the odds of 30-day mortality. Physicians should consider acting quickly to administer antibiotics with S. aureus coverage to any patient suspected of having septic shock. Future work should investigate the relationship between the timing of antibiotics and morality with other pathogens such as gram negative bacteria.

\section{References}

1. Fleischmann C, Scherag A, Adhikari NK, et al. Assessment of Global Incidence and Mortality of Hospital-treated Sepsis. Current Estimates and Limitations. Am Journal Resp Crit Care Med 2016;193:25972

2. Rhodes A, Evans LE, Alhazzani W, et al. Surviving Sepsis Campaign: International Guidelines for Management of Sepsis and Septic Shock: 2016. Intensive Care Med 2017;43:304-77

3. National Health Foundation. NQF endorsed voluntary consensus standards for hospital care: version 5.0a [Internet]. Los Angeles (CA): NHF; cited 2019. Available from: https://www.nhfca.org/psf/ 
resources/Updates1/SEP-1\%20Measure\%20Information\%20Form\%20(MIF).pdf

4. Levy MM, Evans LE, Rhodes A. The Surviving Sepsis Campaign Bundle: 2018 update. Intensive Care Med 2018;44:925-8

5. Kumar A, Roberts D, Wood KE, et al. Duration of hypotension before initiation of effective antimicrobial therapy is the critical determinant of survival in human septic shock. Crit Care Med 2006;34:1589-96

6. Bloos F, Thomas-Ruddel D, Ruddel H, et al. Impact of compliance with infection management guidelines on outcome in patients with severe sepsis: a prospective observational multi-center study. Crit care 2014;18:R42

7. Ferrer R, Martin-Loeches I, Phillips G, et al. Empiric antibiotic treatment reduces mortality in severe sepsis and septic shock from the first hour: results from a guideline-based performance improvement program. Crit Care Med 2014;42:1749-55

8. Gaieski DF, Mikkelsen ME, Band RA, et al. Impact of time to antibiotics on survival in patients with severe sepsis or septic shock in whom early goal-directed therapy was initiated in the emergency department. Crit Care Med 2010;38:1045-53

9. Joo YM, Chae MK, Hwang SY, et al. Impact of timely antibiotic administration on outcomes in patients with severe sepsis and septic shock in the emergency department. Clin Exp Emerg Med 2014;1:3540

10. Puskarich MA, Trzeciak S, Shapiro NI, et al. Association between timing of antibiotic administration and mortality from septic shock in patients treated with a quantitative resuscitation protocol. Crit Care Med 2011;39:2066-71

11. Ryoo SM, Kim WY, Sohn CH, et al. Prognostic value of timing of antibiotic administration in patients with septic shock treated with early quantitative resuscitation Am J Med Sci 2015;349:328-33 
12. Vilella AL, Seifert CF. Timing and appropriateness of initial antibiotic therapy in newly presenting septic patients. Am J of Emerg Med 2014;32:7-13

13. Yokota PK, Marra AR, Martino MD, et al. Impact of appropriate antimicrobial therapy for patients with severe sepsis and septic shock--a quality improvement study. PloS one 2014;9:e104475

14. Sterling SA, Miller WR, Pryor J, Puskarich MA, Jones AE. The Impact of Timing of Antibiotics on Outcomes in Severe Sepsis and Septic Shock: A Systematic Review and Meta-Analysis. Crit Care Med 2015;43:1907-15

15. Wisdom A, Eaton V, Gordon D, Daniel S, Woodman R, Phillips C. INITIAT-E.D.: Impact of timing of INITIation of Antibiotic Therapy on mortality of patients presenting to an Emergency Department with sepsis. Emerg Med Australas 2015;27:196-201

16. Levy MM, Rhodes A, Phillips GS, et al. Surviving Sepsis Campaign: association between performance metrics and outcomes in a 7.5-year study. Intensive Care Med 2014;40:1623-33

17. Karvellas CJ, Abraldes JG, Arabi YM, Kumar A. Appropriate and timely antimicrobial therapy in cirrhotic patients with spontaneous bacterial peritonitis-associated septic shock: a retrospective cohort study. Aliment Pharmacol Ther 2015;41:747-57

18. De Groot B, Ansems A, Gerling DH, et al. The association between time to antibiotics and relevant clinical outcomes in emergency department patients with various stages of sepsis: a prospective multi-center study. Crit care 2015;19:194

19. Burnham JP, Lane MA, Kollef MH. Impact of Sepsis Classification and Multidrug-Resistance Status on Outcome Among Patients Treated With Appropriate Therapy. Crit Care Med 2015;43:1580-6

20. Liu VX, Fielding-Singh V, Greene JD, et al. The Timing of Early Antibiotics and Hospital Mortality in Sepsis. Am Journal Resp Crit Care Med 2017;196:856-63

21. Seymour CW, Gesten F, Prescott HC, et al. Time to Treatment and Mortality during Mandated Emergency Care for Sepsis. N Engl J Med 2017;376:2235-44 
22. Tong SY, Davis JS, Eichenberger E, Holland TL, Fowler VG, Jr. Staphylococcus aureus infections: epidemiology, pathophysiology, clinical manifestations, and management. Clin Microbiol Rev 2015;28:60361

23. Vincent JL, Sakr Y, Sprung CL, et al. Sepsis in European intensive care units: results of the SOAP study. Crit Care Med 2006;34:344-53

24. Hanberger $\mathrm{H}$, Walther S, Leone M, et al. Increased mortality associated with methicillin-resistant Staphylococcus aureus (MRSA) infection in the intensive care unit: results from the EPIC II study. Int $J$ Antimicrob Agents 2011;38:331-5

25. Blot SI, Vandewoude KH, Hoste EA, Colardyn FA. Outcome and attributable mortality in critically Ill patients with bacteremia involving methicillin-susceptible and methicillin-resistant Staphylococcus aureus. Arch Intern Med 2002;162:2229-35

26. Levy MM, Fink MP, Marshall JC, et al. 2001 SCCM/ESICM/ACCP/ATS/SIS International Sepsis Definitions Conference. Crit Care Med 2003;31:1250-6

27. Elixhauser A, Steiner C, Palmer L. Clinical Classifications Software (CCS). US Agency for Healthcare Research and Quality, 2015. Available from: https://www.hcup us.ahrq.gov/toolssoftware/ccs/CCSUsersGuide.pdf

28. Vassar M, Holzmann M. The retrospective chart review: important methodological considerations. $J$ Educ Eval Health Prof 2013;10:12

29. Kadri SS, Rhee C, Strich JR, et al. Estimating Ten-Year Trends in Septic Shock Incidence and Mortality in United States Academic Medical Centers Using Clinical Data. Chest 2017;151:278-85 30. Paul M, Kariv G, Goldberg E, et al. Importance of appropriate empirical antibiotic therapy for methicillin-resistant Staphylococcus aureus bacteraemia. J Antimicrob Chemother 2010;65:2658-65 31. Zelenitsky S, Rubinstein E, Ariano R, et al. Vancomycin pharmacodynamics and survival in patients with methicillin-resistant Staphylococcus aureus-associated septic shock. Int J Antimicrob Agents 
32. Buchan BW, Allen S, Burnham CA, et al. Comparison of the next-generation Xpert MRSA/SA BC assay and the GeneOhm StaphSR assay to routine culture for identification of Staphylococcus aureus and methicillin-resistant S. aureus in positive-blood-culture broths. J Clin Microbiol 2015;53:804-9

33. Pruinelli L, Westra BL, Yadav P, et al. Delay Within the 3-Hour Surviving Sepsis Campaign Guideline on Mortality for Patients With Severe Sepsis and Septic Shock. Crit Care Med 2018;46:500-5.

34. Ventola CL. The antibiotic resistance crisis: part 1: causes and threats. P T 2015;40:277-83

35. Pines JM, Hollander JE, Lee H, Everett WW, Uscher-Pines L, Metlay JP. Emergency department operational changes in response to pay-for-performance and antibiotic timing in pneumonia. Acad Emerg Med 2007; 14:545-8

36. Klompas M, Calandra T, Singer M. Antibiotics for Sepsis-Finding the Equilibrium. Jama 2018;320:1433-4

37. Humphreys H, Fitzpatick F, Harvey BJ. Gender differences in rates of carriage and bloodstream infection caused by methicillin-resistant Staphylococcus aureus: are they real, do they matter and why? Clin Infect Dis 2015;61:1708-14

38. Nachtigall I, Tafelski S, Rothbart A, et al. Gender-related outcome difference is related to course of sepsis on mixed ICUs: a prospective, observational clinical study. Crit Care 2011;15:R151

39. Pietropaoli AP, Glance LG, Oakes D, Fisher SG. Gender differences in mortality in patients with severe sepsis or septic shock. Gend Med 2010;7:422-37

40. Angele MK, Pratschke S, Hubbard WJ, Chaudry IH. Gender differences in sepsis: cardiovascular and immunological aspects. Virulence 2014;5:12-9

41. Kaukonen KM, Bailey M, Suzuki S, Pilcher D, Bellomo R. Mortality related to severe sepsis and septic shock among critically ill patients in Australia and New Zealand, 2000-2012. Jama 2014;311:1308- 
42. Lam SW, Bauer SR, Neuner EA. Predictors of septic shock in patients with methicillin-resistant Staphylococcus aureus bacteremia. Int J Infect Dis 2012;16:e453-6

43. Jolley RJ, Sawka KJ, Yergens DW, Quan H, Jette N, Doig CJ. Validity of administrative data in recording sepsis: a systematic review. Crit Care 2015;19:139

44. Ramanathan R, Leavell P, Stockslager G, Mays C, Harvey D, Duane TM. Validity of International Classification of Diseases, Ninth Revision, Clinical Modification (ICD-9-CM) screening for sepsis in surgical mortalities. Surg Infect 2014;15:513-6

45. Schneeweiss S, Robicsek A, Scranton R, Zuckerman D, Solomon DH. Veteran's affairs hospital discharge databases coded serious bacterial infections accurately. J Clin Epidemiol. 2007;60:397-409

\section{Figure Legends:}

Figure \#1 Participant enrollment diagram.

Figure \#2 Kernel density plot showing time to antibiotic administration from emergency department triage. Figure \#3 Odds ratios of 30-day mortality and the associated 95\% confidence interval for time to first appropriate antibiotic administration. Multivariate model adjusted for: BMI, APACHE III score, infective arthritis, SIRS criteria present at the time of blood culture, and MRSA/MSSA, and continuous time. 
